\title{
Portfolio choice and optimal hedging with general risk functions: A simplex-like algorithm
}

\author{
Alejandro Balbás ${ }^{\mathrm{a}, *}$, Raquel Balbás ${ }^{\mathrm{b}}$, Silvia Mayoral ${ }^{\mathrm{c}}$ \\ a Universidad Carlos III, CL, Madrid 126, 28903 Getafe, Madrid, Spain

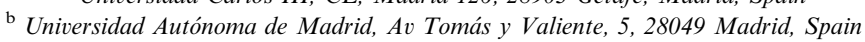 \\ ${ }^{\mathrm{c}}$ Universidad de Navarra, Edificio Bibliotecas, 31080 Pamplona, Navarra, Spain
}

\begin{abstract}
The minimization of general risk functions is becoming more and more important in portfolio choice theory and optimal hedging. There are two major reasons. Firstly, heavy tails and the lack of symmetry in the returns of many assets provokes that the classical opti mization of the standard deviation may lead to dominated strategies, from the point of view of the second order stochastic dominance. Secondly, but not less important, many institutional investors must respect legal capital requirements, which may be more easily studied if one deals with a risk measure related to capital losses.

This paper proposes a new method to simultaneously minimize several general risk or dispersion measures. The representation the orems of risk functions are applied to transform the general risk minimization problem in a minimax problem, and later in a linear pro gramming problem between infinite dimensional Banach spaces. Then, new necessary and sufficient optimality conditions are stated and a simplex like algorithm is developed. The algorithm solves the dual problem and provides both optimal portfolios and their sensitivities.

The approach is general enough and does not depend on any particular risk measure, but some of the most important cases are spe cially analyzed. A final real data numerical example illustrates the practical performance of the proposed methodology.
\end{abstract}

Keywords: Risk measure; Deviation measure; Portfolio selection; Infinite dimensional linear programming; Simplex like method

\section{Introduction}

Modern risk analysis must face two major drawbacks affecting most of the available securities and many investment strategies: asymmetric returns and fat tails. They recently caused important capital losses, that were difficult to predict when agents were using classical risk functions like the standard deviation or sensitivities of the portfolio value with regard to economic or financial variables. Accordingly, regulators have extended the analysis of the group G-10 and the Basle Committee on Banking Supervision, that provided a minimal set of capital requirements in 1988. Besides, many practitioners have also extended those methods of J.P. Morgan during the late 1980s, that developed a firm-wide VaR system in order to predict possible losses under negative scenarios and normal distributions.

Researchers have also focused on this problem and Artzner et al. (1999) published a seminal paper introducing the notion of "coherent measure of risk". They attempted to establish a minimal set of axioms that a risk measure should satisfy in order to adequately reflect capital requirements, and they derived important properties and representation theorems from the axioms. Their analysis has been extended or modified by many authors. Important contributions are, amongst

\footnotetext{
Corresponding author. Tel.: +34 916249636; fax: +34 916249607.

E mail addresses: alejandro.balbas@uc3m.es (A. Balbás), smayoral@unav.es (S. Mayoral).
} 
many others, Rockafellar et al. (2006a,b), where the concepts or "expectation bounded risk measure" and "deviation risk measure" are introduced, studied, related and applied to some portfolio choice linked problems, or Ruszczynski and Shapiro (2007), where interesting portfolio optimization topics are addressed too.

Another line of research focuses on those risk functions compatible with the second order stochastic dominance. For instance, Ogryczak and Ruszczynski (1999) point out that the standard deviation does not satisfy this condition if asymmetric returns are involved, whereas the absolute deviation and semi-deviation really do. This justifies that many authors consider the absolute deviation in portfolio selection problems. For instance, Konno et al. (2005), who can reduce the optimization problem to a linear one because they consider discrete return distributions (generated from recent samples).

Finally, recent literature has also optimized modern risk functions in order to price and hedge in incomplete or imperfect markets. Interesting papers are Föllmer and Schied (2002) and Nakano (2004), among others.

The optimization of dispersions or risk functions reflecting capital requirements is often complex. Indeed, as pointed out by many papers, one frequently must deal with a non-differentiable problem. Though it is usually convex, the subgradientlinked optimality conditions are not so easily treated in practice. Therefore, authors usually look for an equivalent alternative optimization problem. For instance, this is done by Föllmer and Schied (2002) and Nakano (2004). As said above, Konno et al. (2005) can deal with a linear problem, but they minimize a particular dispersion and involve discrete returns. Benati (2003) also uses discrete sample-linked returns and gets other linear problem (that he combines with an integer fractional problem) to optimize the "worst conditional expectation", a coherent measure introduced in Artzner et al. (1999).

The present article proposes a linear programming approach that applies for every risk function satisfying quite general conditions. This is a major difference with respect to the analyses of Benati (2003) or Konno et al. (2005), since their constructions only apply for a specific risk measure. On the contrary we can include many coherent risk measures, and every expectation bounded or deviation measure.

The two crucial keys of our study are the representation theorems of risk measures and the use of Banach spaces to address those problems with infinitely many constraints. The representation theorems allow us to transform a general portfolio choice problem into a minimax problem. Minimax problems are usual in finance and often lead to a linear problem (see, for instance, Young, 1998), but there are cases for which there are no equivalent linear formulations (Barber and Copper, 1998). Here we follow an idea of Balbás and Romera (2007), since these authors transform a minimax problem, providing hedging strategies against the interest rate risk, into an infinite-dimensional linear programming problem between Banach spaces.

Our linear programming approach yields new optimality conditions and algorithms. So, the paper outline is as follows. Section 2 presents the basic notations and assumptions, as well as a general portfolio choice (or hedging) vector optimization problem that becomes a minimax one. Section 3 transforms the minimax problem into a dual couple of linear ones between Banach spaces. The involved dual Banach spaces are $\mathscr{C}(\Delta)$ and $\mathscr{M}(\Delta)$, continuous functions and inner regular Borel measures on the compact space $\Delta$. Theorem 2, the most important result in this section, provides necessary and sufficient optimality conditions that show important differences with respect to those of previous literature. In fact, rather than subgradient-linked properties, we draw on the existence of probability measures satisfying appropriate requirements, in the line of the complementary slackness conditions of linear programming. Section 4 focuses on special optimal hedging or portfolio choice problems that consider a finite number of available assets in the market. The linear problems become semi-infinite, which makes it easier to formulate the optimality conditions of Theorem 2. Section 5 characterizes the extreme points of the dual problem (Theorem 7) and develops a simplex-like algorithm to solve it. The complementary slackness conditions allow us to obtain the primal solution (optimal portfolio). Thus we have both the optimal portfolio and its sensitivity with respect to the involved parameters. Section 6 particularizes the theory for several risk functions, with special focus on some classical ones and those that can be given by distorting functions (Wang, 2000). These risk functions allow us to construct "alternative indexes", whose combinations with the riskless asset lead to the efficient portfolios in a new framework such that risk levels are measured taking into account asymmetries and fat tails. Section 7 deals with a real data numerical example that illustrates the performance of our methods in practice. Despite the linear optimization problem involves infinite-dimensional spaces, the objective function only depends on a finite-dimensional subspace, so the convergence of the algorithm is really fast. Section 8 concludes the paper.

\section{Preliminaries and notations}

Let $(\Omega, \mathscr{F}, \mu)$ be a probability space, $p \in[1, \infty)$ and $q \in(1, \infty]$ such that $(1 / p)+(1 / q) \quad 1$, where, as usual, we take the convention $(1 / \infty) \quad 0$. It is well known that $L^{q}=L^{q}(\Omega, \mathscr{F}, \mu)$ is the dual space of $L^{P}$. Consider the functions $\rho_{i}: L^{p} \mapsto \mathbb{R}$, $i \quad 1,2, \ldots, \gamma$, given by

$$
\rho_{i}(y)=\operatorname{Sup}\left\{-E(y z): z \in \Delta_{i}\right\}
$$

each $\Delta_{i}$ being a non-void convex and $\sigma\left(L^{q}, L^{p}\right) \quad$ compact subset of $L^{q}$ and 


$$
E(h)=\int_{\Omega} h(\omega) \mathrm{d} \mu(\omega)=\int_{\Omega} h \mathrm{~d} \mu
$$

denoting the mathematical expectation of every random variable $h \in L^{1}$. Notice that $L^{q} \ni z \mapsto E(y z) \in \mathbb{R}$ is $\sigma\left(L^{q}, L^{p}\right) \quad$ continuous for every $y \in L^{p}$ and, therefore, Sup may be replaced by Max in (1).

Many coherent risk measures, in the sense of Artzner et al. (1999), or expectation bounded risk measures, in the sense of Rockafellar et al. (2006a), satisfy expression (1), as pointed out in Rockafellar et al. (2006a,b). Actually, these authors show that $\Delta_{i}$ is $\sigma\left(L^{q}, L^{p}\right)$-closed because they allow for risk functions whose value may equal $+\infty$. However, owing to the Alaoglu's Theorem (see Holmes, 1975), the $\sigma\left(L^{q}, L^{p}\right)$-compactness of $\Delta_{i}$ will hold if $\rho_{i}$ is a continuous $\mathbb{R}$-valued expectation bounded risk measure (see Section 6 and the Representation Theorems of convex functions in Zalinescu, 2002).

Besides, Rockafellar et al. (2006a) introduce the notion of deviation measure $D$ and they show that

$$
D(y)=R(y)+E(y), \quad \forall y \in L^{p},
$$

$R$ denoting an expectation bounded risk measure. Obviously, since $R$ satisfies (1) so does $D$ once $\Delta_{i}$ is substituted by

$$
\Delta_{i}-\{1\}=\left\{z-1 ; z \in \Delta_{i}\right\} .
$$

Whence, our analysis may apply for both risk and deviation functions.

Fix $m \in \mathbb{N}$, a set of real numbers $\left\{b_{j}\right\}_{j}^{m}$ and a set of random variables $\left\{q_{j}\right\}_{j}^{m} \subset L^{q}$. We will deal with the vector optimization problem:

$$
\left.\begin{array}{ll}
\operatorname{Min} & \rho(y) \\
& E\left(y q_{j}\right) \leqslant b_{j}, \quad j=1,2, \ldots, m \\
& y \in Y
\end{array}\right\} .
$$

$Y \subset L^{p}$ being an arbitrary convex cone, and $\rho: L^{P} \mapsto \mathbb{R}^{\gamma}$ denoting the vector function with components $\rho_{i}, i \quad 1,2, \ldots, \gamma \cdot(4)$ may be understood as a portfolio selection or an optimal hedging problem such that there is no a clear agreement about the most convenient way to measure the risk level. Constraints $E\left(y q_{j}\right) \leqslant b_{j}$ (or, equivalently, $\left.-E\left(y q_{j}\right) \geqslant-b_{j}\right)$ may reflect several practical situations. For example, a maximum amount of money to invest, a minimum required expected pay-off, etc.

Expression (1) points out that $\rho$ is a convex function and, consequently, for every optimal solution $y_{0}$ of (4) there exists $\alpha \quad\left(\alpha_{1}, \alpha_{2}, \ldots, \alpha_{\gamma}\right)$ such that $\alpha \neq 0, \alpha \geqslant 0$ and $y_{0}$ solves the scalar optimization problem

$$
\left.\operatorname{Min} \begin{array}{l}
\sum_{i=1}^{\gamma} \alpha_{i} \rho_{i}(y) \\
E\left(y q_{j}\right) \leqslant b_{j}, \quad j=1,2, \ldots, m \\
y \in Y
\end{array}\right\} .
$$

Conversely, if $\alpha_{i}>0, i \quad 1,2, \ldots, \gamma$, then every solution of (5) also solves (4). Thus, by solving problem (5), with $\alpha$ arbitrary, we will get the whole set of minimal solutions of (4) and, possibly, some more points. All of them compose the so called "set of weak solutions of (4)". Henceforth we will fix a non-null and non-negative arbitrary vector $\alpha$, so as to obtain those weak solutions.

\section{Minimizing risk measures by linear optimization in Banach spaces: Optimality conditions}

In this section we will present several problems whose solution leads to the solution of problem (5). Furthermore, most of the yielded alternative problems will be linear, which will allow us to characterize their solutions by means of duality relationships and complementary slackness conditions. The dual variables generate economic interpretations applying in practical portfolio choice problems. Finally, a future section will provide a simplex-like algorithm permitting us to solve the dual (and therefore the primal) problem.

Denote by $\Delta \quad \Delta_{1} \times \Delta_{2} \times \cdots \times \Delta_{\gamma}$ the usual product of $\left(\Delta_{i}\right)_{i}^{\gamma}$ endowed with the product of the weak ${ }^{*}$-topologies. Then $\Delta$ is obviously convex and compact.

Consider problem

$$
\left.\begin{array}{ll}
\text { Min } & \theta \\
& \theta+\sum_{i 1}^{\gamma} \alpha_{i} E\left(y z_{i}\right) \geqslant 0, \quad \forall z=\left(z_{1}, z_{2}, \ldots, z_{\gamma}\right) \in \Delta \\
& E\left(y q_{j}\right) \leqslant b_{j}, \quad j=1,2, \ldots, m \\
& \theta \in \mathbb{R}, \quad y \in Y
\end{array}\right\} .
$$

$(\theta, y) \in \mathbb{R} \times L^{p}$ being the decision variable. We have the following result whose proof is very simple and therefore omitted. 
Proposition 1. Suppose that $\theta_{0} \in \mathbb{R}$ and $y_{0} \in Y$. Then, $y_{0}$ solves (5) and

$$
\theta_{0}=\sum_{i=1}^{\gamma} \alpha_{i} \rho_{i}\left(y_{0}\right)
$$

if and only if $\left(\theta_{0}, y_{0}\right)$ solves $(6){ }^{1}$

Remark 1. Problem (6) is equivalent to problem

$$
\left.\begin{array}{ll}
\text { Min } & \theta \\
& \theta+E(y z) \geqslant 0, \quad \forall z \in \Delta^{\alpha} \\
& E\left(y q_{j}\right) \leqslant b_{j}, \quad j=1,2, \ldots, m \\
& \theta \in \mathbb{R}, \quad y \in Y
\end{array}\right\}
$$

$\Delta^{\alpha}$ being the convex and $\sigma\left(L^{q}, L^{p}\right)$ - compact subset of $L^{q}$ given by

$$
\Delta^{\alpha}=\sum_{i}^{\gamma} \alpha_{i} \Delta_{i}=\left\{z \in L^{q} ; z=\sum_{i}^{\gamma} \alpha_{i} z_{i}, z_{i} \in \Delta_{i}, i=1,2, \ldots, \gamma\right\} .
$$

This new formulation would simplify the notation of future analyses, although we will focus on problem (6) because it might be complicated to determine $\Delta^{\alpha}$ in practical examples.

Notice that problem (6) is linear and its first constraint is established in the Banach space $\mathscr{C}(\Delta)$ of real valued and continuous functions on the compact space $\Delta$ endowed with the supremum norm. According to the Riesz Representation Theorem, its dual space, denoted by $\mathscr{M}(\Delta)$, is composed of the real valued inner regular and $\sigma-$ additive measures on the Borel $\sigma$-algebra of $\Delta$ endowed with the norm of the total variation.

The Lagrangian function

$$
\mathscr{L}: \mathbb{R} \times L^{p} \times \mathbb{R}^{m} \times \mathscr{M}(\Delta) \mapsto \mathbb{R}
$$

of (6) becomes

$$
\begin{aligned}
\mathscr{L}(\theta, y, \lambda, v) & =\theta-\int_{\Delta}\left(\theta+\sum_{i 1}^{\gamma} \alpha_{i} E\left(y z_{i}\right)\right) \mathrm{d} v(z)+\sum_{j 1}^{m} \lambda_{j}\left(E\left(y q_{j}\right)-b_{j}\right) \\
& =\theta(1-v(\Delta))-\sum_{i 1}^{\gamma} \alpha_{i}\left(\int_{\Delta} E\left(y z_{i}\right) \mathrm{d} v(z)\right)+\sum_{j 1}^{m} \lambda_{j}\left(E\left(y q_{j}\right)-b_{j}\right) .
\end{aligned}
$$

In order to simplify some expressions, if convenient we will represent by $v_{i} \pi_{i}(v)$ the standard projection of $v$ on $\Delta_{i}$, $i \quad 1,2, \ldots, \gamma$, and it is obvious that

$$
\int_{\Delta} E\left(y z_{i}\right) \mathrm{d} v(z)=\int_{\Delta_{i}} E\left(y z_{i}\right) \mathrm{d} v_{i}\left(z_{i}\right)
$$

$i \quad 1,2, \ldots, \gamma$. Moreover, the integration variables $z$ or $z_{i}$ may be omitted.

According to Anderson and Nash (1987), a couple $(\lambda, v) \in \mathbb{R}^{m} \times \mathscr{M}(\Delta)$ is dual feasible for Problem (6) if and only if $\lambda \geqslant 0, v \geqslant 0$ and

$$
\operatorname{Inf}\left\{\theta(1-v(\Delta))-\sum_{i=1}^{\gamma} \alpha_{i}\left(\int_{\Delta} E\left(y z_{i}\right) \mathrm{d} v\right)+\sum_{j=1}^{m} \lambda_{j}\left(E\left(y q_{j}\right)-b_{j}\right) ;(\theta, y) \in \mathbb{R} \times Y\right\}>-\infty,
$$

in which case its dual objective value is given by the infimum above. Thus, the dual problem becomes

\footnotetext{
${ }^{1}$ Among others, Shimizu and Aiyoshy (1980) already introduced a new variable in order to simplify a minimax problem. The proposition above just applies this idea.
} 


$$
\left.\begin{array}{ll}
\operatorname{Max} & -\sum_{j 1}^{m} b_{j} \lambda_{j} \\
& \sum_{j 1}^{m} \lambda_{j} E\left(y q_{j}\right)-\sum_{i 1}^{\gamma} \alpha_{i}\left(\int_{\Delta} E\left(y z_{i}\right) \mathrm{d} v\right) \geqslant 0, \quad \forall y \in Y \\
& v(\Delta)=1 \\
& (\lambda, v) \in \mathbb{R}^{m} \times \mathscr{M}(\Delta) \\
& \lambda \geqslant 0, \quad v \geqslant 0
\end{array}\right\} .
$$

$(\lambda, v) \in \mathbb{R}^{m} \times \mathscr{M}(\Delta)$ being the decision variable.

It is trivial to see that the inequality

$$
\theta \geqslant-\sum_{j=1}^{m} b_{j} \lambda_{j}
$$

holds whenever $(\theta, y)$ and $(\lambda, v)$ are (6) and (8)-feasible respectively.

Since (6) and (8) involve infinite-dimensional spaces the absence of duality gap is not guaranteed. To solve this minor drawback we will impose the usual Slater Qualification (see Anderson and Nash, 1987). Furthermore, in practical examples it will not be realistic to assume that the risk level may tend to $-\infty$. Thus, we will consider that (6) is bounded.

Assumption 1. There exists $y_{0} \in Y$ such that $E\left(y_{0} q_{j}\right)<b_{j}, j \quad 1,2, \ldots, m$. Moreover, there exists $\theta^{*}$ such that $\theta \geqslant \theta^{*}$ whenever $(\theta, y)$ is $(6)$-feasible.

The latter assumption implies that (6) has a finite infimum value and (8) attains its optimal value, i.e., (8) is solvable. We will denote by $\theta_{\alpha}$ the optimal value of both problems.

The second and the last constraints in (8) reveal that (8)-feasible inner regular measures $v$ have to be probabilities. Hereafter we will denote

$$
\mathscr{P}(\Delta)=\{v \in \mathscr{M}(\Delta) ; v \geqslant 0, \quad v(\Delta)=1\} .
$$

The Alaoglu's Theorem easily leads to the compactness of $\mathscr{P}(\Delta)$ when endowed with the $\sigma(\mathscr{M}(\Delta), \mathscr{C}(\Delta))$-topology (Holmes, 1975, or Anderson and Nash, 1987). The same notations and comments apply if $\Delta$ is replaced by $\Delta_{i}, i \quad 1,2, \ldots, \gamma$.

Notice that the influence of the $v$ variable in (8) only depends on $\left\{v_{i}\right\}_{i}^{\gamma}$, in the sense that two probability measures with similar projections have similar role in this optimization problem. Thus, if convenient, the dual variable $v$ may be substituted by its projections $\left\{v_{i}\right\}_{i}^{\gamma}$.

The first constraint of (8) involves the primal variable $y \in L^{p}$. This makes some notations "rather complex", so it is worthwhile to eliminate $y$. Notice that

$$
L^{p} \ni y \mapsto \sum_{i=1}^{\gamma} \alpha_{i}\left(\int_{\Delta_{i}} E\left(y z_{i}\right) \mathrm{d} v_{i}\right) \in \mathbb{R}
$$

is linear and continuous for every $v \in \mathscr{M}(\Delta)$. Thus it may be represented by a vector of $L^{q}$ that we will denote by

$$
\sum_{i=1}^{\gamma} \alpha_{i}\left(\int_{\Delta_{i}} E(-) \mathrm{d} v_{i}\right)
$$

So, in order to remove the $y$ variable from (8) we will draw on the notation

$$
\sum_{j}^{m} q_{j} \lambda_{j}-\sum_{i=1}^{\gamma} \alpha_{i}\left(\int_{\Delta_{i}} E(-) \mathrm{d} v_{i}\right) \geqslant_{Y} 0 .
$$

Then, the dual problem becomes

$$
\left.\begin{array}{l}
\operatorname{Max} \quad-\sum_{j 1}^{m} b_{j} \lambda_{j} \\
\sum_{j 1}^{m} q_{j} \lambda_{j}-\sum_{i 1}^{\gamma} \alpha_{i}\left(\int_{\Delta_{i}} E(-) \mathrm{d} v_{i}\right) \geqslant_{Y} 0 \\
v(\Delta)=1 \\
(\lambda, v) \in \mathbb{R}^{m} \times \mathscr{M}(\Delta) \\
\lambda \geqslant 0, \quad v \geqslant 0
\end{array}\right\}
$$

The absence of duality gap permits us to characterize primal and dual solutions by means of a system of equations and inequalities. 
Theorem 2. Consider $(\theta, y) \in \mathbb{R} \times L^{p}$ and $(\lambda, v) \in \mathbb{R}^{m} \times \mathscr{P}(\Delta)$. They solve (6) and (11) if and only if they solve the following system of complementary slackness conditions

$$
\left.\begin{array}{ll}
\sum_{j 1}^{m} \lambda_{j} E\left(y q_{j}\right)-\sum_{i 1}^{\gamma} \alpha_{i}\left(\int_{\Delta_{i}} E\left(y z_{i}\right) \mathrm{d} v_{i}\right)=0 & \\
\lambda_{j}\left(E\left(y q_{j}\right)-b_{j}\right)=0, & \\
\theta+\sum_{i 1}^{\gamma} \alpha_{i}\left(\int_{\Delta_{i}} E\left(y z_{i}\right) \mathrm{d} v_{i}\right)=0 & \\
\theta+\sum_{i 1}^{\gamma} \alpha_{i} E\left(y z_{i}\right) \geqslant 0, & \\
E\left(y q_{j}\right) \leqslant b_{j}, & \\
\sum_{j}^{m} q_{j} \lambda_{j}-\sum_{i 1}^{\gamma} \alpha_{i}\left(\int_{\Delta_{i}} E(-) \mathrm{d} v_{i}\right) \geqslant_{Y} 0 & \\
y \in Y, \quad \lambda \geqslant 0 &
\end{array}\right\}
$$

Proof. The four inequalities of the system above point out that both $(\theta, z)$ and $(\lambda, v)$ are feasible. Besides, the first equality gives

$$
-\sum_{j 1}^{m} \lambda_{j} E\left(y q_{j}\right)=-\sum_{i=1}^{\gamma} \alpha_{i}\left(\int_{\Delta_{i}} E\left(y z_{i}\right) \mathrm{d} v_{i}\right) .
$$

From the second equality,

$$
-\sum_{j=1}^{m} \lambda_{j} b_{j}=-\sum_{i=1}^{\gamma} \alpha_{i}\left(\int_{\Delta_{i}} E\left(y z_{i}\right) \mathrm{d} v_{i}\right) .
$$

From the third equality,

$$
-\sum_{j=1}^{m} \lambda_{j} b_{j}=\theta
$$

and (9) shows that we are facing the solutions of both problems.

Conversely, suppose that $(\theta, y)$ and $(\lambda, v)$ solve both problems. Then they must be feasible and hence they must satisfy the four inequalities. On the other hand, the complementary slackness conditions of linear programming (Anderson and Nash, 1987) lead to the second and third expressions of (12). Finally, $(\theta, y)$ must minimize the Lagrangian Function (7) on $\mathbb{R} \times Y$ (Anderson and Nash, 1987). Since the dual constrains trivially show that the minimal value of $(7)$ is attained at $(0,0)$ and reaches the value $-\sum_{j=1}^{m} \lambda_{j} b_{j}$, we easily obtain the first equality of (12).

Remark 2. System (12) provides necessary and sufficient optimality conditions for problems (5) and (6) that do not use the subgradients of the risk vector measure $\rho$. On the contrary, these conditions draw on the existence of inner regular probability measures $\left\{v_{i}\right\}_{i 1}^{\gamma}$ on $\left\{\Delta_{i}\right\}_{i}^{\gamma}$ i whose supports are contained on the zeros of

$$
\theta+\sum_{i=1}^{\gamma} \alpha_{i} E\left(y z_{i}\right)
$$

and multipliers $\left\{\lambda_{j}\right\}_{j 1}^{m}$ such that (10) holds. This fact may be an interesting alternative to solve many portfolio choice or optimal hedging problems in practice, although it will not be easy to achieve the explicit solution of System (12). ${ }^{2}$ However, to deal with the system becomes far easier if the dual solution may be computed by means of alternative procedures. This is the reason why in Section 5 we will develop a simplex-like method for the dual problem that applies for those cases such that the convex cone $Y$ is generated by a finite set of available securities.

\footnotetext{
$\overline{2}$ Recall that dealing with subgradients may also generate significant problems in practice. See Rockafellar et al. (2006a) or Ruszczynski and Shapiro (2007) for complementary analyses.
} 


\section{Semi-infinite problems}

In this section we will consider $n=r+s \in \mathbb{N}$ available assets whose pay-offs are $\left\{y_{h}\right\}_{h}^{n} \subset L^{p}$. Strategies will be represented by $x \in \mathbb{R}^{n}$, and the closed convex cone

$$
X=\left\{x=\left(x_{h}\right)_{h 1}^{r+s} \in \mathbb{R}^{r+s} ; x_{h} \geqslant 0, h=r+1, r+2, \ldots, r+s\right\}
$$

will reflect that short-sales of some securities are not allowed. ${ }^{3}$

The set $Y$ (of reachable pay-offs) will be given by

$$
Y=\left\{y \in L^{p} ; y=\sum_{h 1}^{n} x_{h} y_{h}, x=\left(x_{h}\right)_{h 1}^{n} \in X\right\}
$$

It is easy to verify that $Y$ is a convex cone.

Problem (6) becomes

$$
\begin{aligned}
& \text { Min } \theta \\
& \left.\begin{array}{ll}
\theta+\sum_{h 11}^{n}\left(\sum_{i 1}^{\gamma} \alpha_{i} E\left(y_{h} z_{i}\right)\right) x_{h} \geqslant 0, & \forall z \in \Delta \\
\sum_{h 1}^{n} E\left(y_{h} q_{j}\right) x_{h} \leqslant b_{j}, & j=1,2, \ldots, m \\
\theta \in \mathbb{R}, x_{h} \geqslant 0 & h=r+1, \ldots, r+s
\end{array}\right\} .
\end{aligned}
$$

$(\theta, x) \in \mathbb{R}^{1+n}$ being the decision variable. Notice that linear constraints with the form $\sum_{h}^{n}{ }_{1} l_{h} x_{h} \leqslant L$ are easily incorporated in (13). Indeed, take $\tilde{q} \in L^{q}$ such that $E\left(y_{h} \tilde{q}\right)=l_{h}, h \quad 1,2, \ldots, n$, and apply the second constraint in (13). Furthermore, the existence of $\tilde{q}$ holds under weak conditions like the linear independence of $\left\{y_{h}\right\}_{h}^{n}$. In order to prevent the existence of duality gaps we will still assume the fulfillment of the Slater Qualification along with the existence of primal lower bounds (Assumption 1).

In order to adapt the dual problem (11) we need to analyze the constraint (10). Manipulating we have

$$
\sum_{h 1}^{n} E\left[\left(\sum_{j=1}^{m} q_{j} \lambda_{j}\right) y_{h}\right] x_{h} \geqslant \sum_{h 1}^{n}\left[\sum_{i=1}^{\gamma} \alpha_{i}\left(\int_{\Delta_{i}} E\left(y_{h} z_{i}\right) \mathrm{d} v_{i}\right)\right] x_{h}
$$

for every $x \in X$. Then, (11) obviously becomes

\footnotetext{
${ }^{3}$ The cone $X$ above may be replaced by a more general convex closed cone $\widetilde{X} \subset \mathbb{R}^{n}$. Then, the Representation Theorem of closed convex subsets of $\mathbb{R}^{n}$ (Holmes, 1975) shows that $\widetilde{X} \quad L_{\tilde{X}}+C_{\tilde{X}}, L_{\tilde{X}}$ being the vector space.

$$
L_{X} \quad\left\{x \in \mathbb{R}^{n} ; x+\tilde{X} \quad \tilde{X}\right\},
$$

and $C_{X}$ being the closed pointed convex cone (or recession cone)$$
C_{X} \quad\left\{x \in \mathbb{R}^{n} ; x+\left(\tilde{X} \cap L_{X}\right) \subset \tilde{X} \cap L_{X}\right\},
$$

$L_{X}$ representing the orthogonal subspace of $L_{X}$. In the most important cases one has that

$$
L_{X} \quad \mathbb{R}^{n} \quad \text { and } \quad C_{X} \quad\{0\}
$$

for $\tilde{X} \quad \mathbb{R}^{n}$ and

$$
L_{X} \quad\{0\} \quad \text { and } \quad C_{X} \quad \mathbb{R}_{+}^{n}
$$

for $\tilde{X} \mathbb{R}_{+}^{n}$. More generally,

$$
L_{X} \quad\{0\} \quad \text { and } \quad C_{X} \quad \tilde{X}
$$

if $\widetilde{X}$ is pointed. By using the above representation of $\widetilde{X}$ and applying the procedure proposed in Balbás and Romera (2007), we can adapt the variables of our problem so that a $X$ like cone can play the role of $\widetilde{X}$.
} 


$$
\left.\begin{array}{lll}
\operatorname{Max} & -\sum_{j 1}^{m} b_{j} \lambda_{j} \\
& \sum_{j 1}^{m} E\left(q_{j} y_{h}\right) \lambda_{j}=\sum_{i 1}^{\gamma} \alpha_{i}\left(\int_{\Delta_{i}} E\left(y_{h} z_{i}\right) \mathrm{d} v_{i}\right), \quad h=1,2, \ldots, r \\
& \sum_{j 1}^{m} E\left(q_{j} y_{h}\right) \lambda_{j} \geqslant \sum_{i 1}^{\gamma} \alpha_{i}\left(\int_{\Delta_{i}} E\left(y_{h} z_{i}\right) \mathrm{d} v_{i}\right), \quad h=r+1, \ldots, r+s \\
& (\lambda, v) \in \mathbb{R}^{m} \times \mathscr{P}(\Delta) \\
& \lambda \geqslant 0
\end{array}\right\}
$$

$(\lambda, v) \in \mathbb{R}^{m} \times \mathscr{P}(\Delta)$ being the decision variable.

According to Theorem 2, the complementary slackness conditions (12) are necessary and sufficient optimality conditions. In our case they are

$$
\left.\begin{array}{ll}
\sum_{j=1}^{m} \lambda_{j} E\left(y_{h} q_{j}\right)-\sum_{i=1}^{\gamma} \alpha_{i}\left(\int_{\Delta_{i}} E\left(y_{h} z_{i}\right) \mathrm{d} v_{i}\right)=0, & h=1,2, \ldots, r \\
{\left[\sum_{j=1}^{m} \lambda_{j} E\left(y_{h} q_{j}\right)-\sum_{i 1}^{\gamma} \alpha_{i}\left(\int_{\Delta_{i}} E\left(y_{h} z_{i}\right) \mathrm{d} v_{i}\right)\right] x_{h}=0,} & h=r+1, \ldots, r+s \\
\lambda_{j}\left[\sum_{h 1}^{n} E\left(y_{h} q_{j}\right) x_{h}-b_{j}\right]=0, & j=1,2, \ldots, m \\
\theta+\sum_{h 1}^{n}\left[\sum_{i=1}^{\gamma} \alpha_{i}\left(\int_{\Delta_{i}} E\left(y_{h} z_{i}\right) \mathrm{d} v_{i}\right)\right] x_{h}=0 &
\end{array}\right\}
$$

along with the constraints of both problems. As already said, it may be difficult in practice to solve the system above in the unknowns $\theta, x, \lambda$ and $v$. However, if $\lambda$ and $v$ are known, then (15) becomes

$$
\begin{array}{ll}
{\left[\sum_{j=1}^{m} \lambda_{j} E\left(y_{h} q_{j}\right)-\sum_{i=1}^{\gamma} \alpha_{i}\left(\int_{\Delta_{i}} E\left(y_{h} z_{i}\right) \mathrm{d} v_{i}\right)\right] x_{h}=0,} & h=r+1, \ldots, r+s \\
\sum_{h 1}^{n} E\left(y_{h} q_{j}\right) x_{h}=b_{j}, & j=1,2, \ldots, m, \quad \lambda_{j} \neq \\
\theta+\sum_{h 1}^{n}\left[\sum_{i=1}^{\gamma} \alpha_{i}\left(\int_{\Delta_{i}} E\left(y_{h} z_{i}\right) \mathrm{d} v_{i}\right)\right] x_{h}=0 &
\end{array}
$$

which is a simple linear system in $\theta$ and $x$.

\section{The simplex-like algorithm}

This section is devoted to present a simplex-like algorithm so as to solve the semi-infinite linear programming problem (14). Then, (16) will generate the primal solution too.

First of all we will introduce the slackness variables $\left(\xi_{h}\right)_{h r+1}^{r+s}$ in order to get equality constraints. Thus, consider the equivalent problem

$$
\begin{aligned}
& \operatorname{Max}-\sum_{j}^{m} b_{j} \lambda_{j} \\
& \sum_{j=1}^{m} E\left(q_{j} y_{h}\right) \lambda_{j}-\sum_{i 1}^{\gamma} \alpha_{i}\left(\int_{\Delta_{i}} E\left(y_{h} z_{i}\right) \mathrm{d} v_{i}\right)=0, \quad h=1,2, \ldots, r \\
& \left.\sum_{j}^{m} E\left(q_{j} y_{h}\right) \lambda_{j}-\xi_{h}-\sum_{i}^{\gamma} \alpha_{i}\left(\int_{\Delta_{i}} E\left(y_{h} z_{i}\right) \mathrm{d} v_{i}\right)=0, \quad h=r+1, \ldots, r+s\right\} . \\
& (\lambda, \xi, v) \in \mathbb{R}^{m} \times \mathbb{R}^{s} \times \mathscr{P}(\Delta) \\
& \lambda \geqslant 0, \quad \xi \geqslant 0
\end{aligned}
$$

As said above, $\mathscr{P}(\Delta)$ is convex and $\sigma(\mathscr{M}(\Delta), \mathscr{C}(\Delta))$-compact. Besides, given $z \in \Delta$ we will denote by $\delta_{z} \in \mathscr{P}(\Delta)$ the usual Dirac delta that concentrates the mass on $\{z\}$, i.e., $\delta_{z}(\{z\}) \quad 1$ and $\delta_{z}(\Delta \backslash\{z\}) \quad 0$. It is known that the set of extreme points of $\mathscr{P}(\Delta)$ is given by

$$
\operatorname{ext}(\mathscr{P}(\Delta))=\left\{\delta_{z} ; z \in \Delta\right\}
$$

though we will not have to draw on this result. Similar properties hold if $\Delta$ is substituted by $\Delta_{i}, i \quad 1,2, \ldots, \gamma$. 
Lemma 3. Denote by $\mathscr{A}$ the feasible set of (17) and by

$$
\mathscr{A}_{0}=\left\{(\lambda, \xi) \in \mathbb{R}^{m} \times \mathbb{R}^{s} ; \text { there exists } v \in \mathscr{P}(\Delta) \text { with }(\lambda, \xi, v) \in \mathscr{A}\right\} .
$$

Then, $\mathscr{A}_{0}$ is convex and closed.

Proof. It is easy to see that $\mathscr{A}_{0}$ is convex, so let us prove that it is closed. Indeed, take the sequence $\left\{\left(\lambda_{k}, \xi_{k}\right)\right\}_{k}^{\infty} \subset \mathscr{A}_{0}$ and suppose that

$$
\lim _{k \mapsto \infty}\left(\lambda_{k}, \xi_{k}\right)=(\lambda, \xi) .
$$

We must prove that $(\lambda, \xi) \in \mathscr{A}_{0}$. Take $\left\{\left(v_{k}\right)\right\}_{k}^{\infty} \subset \mathscr{P}(\Delta)$ such that $\left(\lambda_{k}, \xi_{k}, v_{k}\right) \in \mathscr{A}$. Since $\mathscr{P}(\Delta)$ is compact there exists $v$, agglomeration point of $\left\{\left(v_{k}\right)\right\}_{k 1}^{\infty}$. Therefore, $(\lambda, \xi, v)$ is an agglomeration point of $\left\{\left(\lambda_{k}, \xi_{k}, v_{k}\right)\right\}_{k 1}^{\infty}$. Since the points of this sequence are in $\mathscr{A}$ and $\mathscr{A}$ is closed, $(\lambda, \xi, v)$ is in $\mathscr{A}$ too. Hence, $(\lambda, \xi) \in \mathscr{A}_{0}$.

Lemma 4. Consider the sets $\mathscr{A}_{0}$ and $\mathscr{A}$ above and their extreme sets, $\operatorname{ext}\left(\mathscr{A}_{0}\right)$ and $\operatorname{ext}(\mathscr{A})$. Then, there exists $(\lambda, \xi, v) \in \operatorname{ext}(\mathscr{A})$ such that $-\sum_{j{ }_{1}}^{m} b_{j} \lambda_{j}=\theta_{\alpha}$, i.e., there exist $(\lambda, \xi, v) \in \operatorname{ext}(\mathscr{A})$ solving $(17)$. Moreover, $(\lambda, \xi) \in \operatorname{ext}\left(\mathscr{A}_{0}\right){ }^{4}$

Proof. The previous lemma shows that $\mathscr{A}_{0}$ is convex and closed. Consequently, the Representation Theorem of closed convex subsets apply (Holmes, 1975). Since $\mathscr{A}_{0}$ is included in the non-negative cone of $\mathbb{R}^{m} \times \square \mathbb{R}^{s}$ it does not contain any affine manifold, and therefore

$$
\mathscr{A}_{0}=\operatorname{Co}\left[\operatorname{ext}\left(\mathscr{A}_{0}\right)\right]+R c\left(\mathscr{A}_{0}\right) \text {, }
$$

Co denoting convex hulls and $R c$ denoting recession cones.

Assumption 1 guarantees that (17) is solvable, and, accordingly, (18) ensures the existence of $(\lambda, \xi) \in \operatorname{ext}\left(\mathscr{A}_{0}\right) \operatorname{such}$ that $-\sum_{j=1}^{m} b_{j} \lambda_{j}=\theta_{\alpha}$. Fix $(\lambda, \xi)$ and set

$$
\mathscr{A}_{1}=\{v \in \mathscr{P}(\Delta) ;(\lambda, \xi, v) \in \mathscr{A}\}
$$

It is easy to check that $\mathscr{A}_{1}$ is closed, and therefore compact because it is included in $\mathscr{P}(\Delta)$. The Krein Milman Theorem (Holmes, 1975, or Anderson and Nash, 1987) ensures that $\mathscr{A}_{1}$ is the $\sigma(\mathscr{M}(\Delta), \mathscr{C}(\Delta))$-closed convex hull of its extreme set. Thus, there exists $v \in \operatorname{ext}\left(\mathscr{A}_{1}\right)$, and $(\lambda, \xi, v)$ is the required element of $\operatorname{ext}(\mathscr{A})$.

Lemma 5. Let be $N \in \mathbb{N}$ and $C \subset \mathbb{R}^{N}$ a convex compact set. Let $c_{0}$ be a extreme point of $C$. Then, there exists a linear map $L: \mathbb{R}^{N} \mapsto \mathbb{R}$ with $L\left(c_{0}\right)>L(c)$ for every $c \in C \backslash\left\{c_{0}\right\}$.

Proof. Let us apply the induction method on the dimension of $C, \operatorname{Dim}(C)$ (i.e., the dimension of the minimal affine manifold containing $C$ ). The result is clear if $\operatorname{Dim}(C)$ equals zero or one. Suppose that it also holds for dimensions $1,2, \ldots, \operatorname{Dim}(C)-1$. Without loss of generality we can assume that $\operatorname{Dim}(C) N$. The Separation Theorems for convex sets (Holmes, 1975) point out the existence of a non-null linear map $L^{\prime}: \mathbb{a} \mathbb{R}^{N} \mapsto \mathbb{R}$ with $L^{\prime}\left(c_{0}\right) \geqslant L^{\prime}(c)$ for every $c \in C$. Obviously,

$$
\operatorname{Dim}\left\{c \in C ; L^{\prime}(c)=L^{\prime}\left(c_{0}\right)\right\} \leqslant N-1
$$

(this set is included in a $(N-1)$-dimensional affine manifold). Due to the induction hypothesis it is easy to establish the existence of $L^{\prime \prime}: \mathbb{R}^{N} \mapsto \mathbb{R}$ with $L^{\prime \prime}\left(c_{0}\right) \geqslant L^{\prime \prime}(c)$ for every $c \in C$ and $L^{\prime \prime}\left(c_{0}\right)>L^{\prime \prime}(c)$ whenever $c \in C$ and $L^{\prime}\left(c_{0}\right) \quad L^{\prime}(c)$. Obviously, $L \quad L^{\prime}+L^{\prime \prime}$ is the required linear map.

Lemma 6. Let be $N \in \mathbb{N}$ and $f: \Delta \mapsto \mathbb{R}^{N}$ a $\sigma\left(L^{q}, L^{p}\right)$-continuous function. Then, for every $v \in \mathscr{P}(\Delta)$ there exist a finite subset

$$
\left\{z^{1}, z^{2}, \ldots, z^{K}\right\} \subset \Delta
$$

and a linear convex combination

$$
\sum_{k}^{K} t_{k} \delta_{z^{k}} t_{1}, t_{2}, \ldots, t_{K} \geqslant 0, \sum_{k=1}^{K} t_{k}=1
$$

\footnotetext{
${ }^{4}$ Given a linear programming problem between infinite dimensional Banach spaces, if the feasible set is weakly compact and the problem is bounded, then there exists a extreme point of the feasible set where the objective function is as close as desired to the optimal value (Anderson and Nash, 1987). However, since our objective does not depend on the infinite dimensional variable $v$, we can prove much more.
} 
such that

$$
\int_{\Delta} f \mathrm{~d} v=\int_{\Delta} f \mathrm{~d}\left(\sum_{k=1}^{K} t_{k} \delta_{z^{k}}\right) .
$$

Proof. Since $f$ is continuous then $f(\Delta)$ is connected and compact. Consider the function

$$
\mathscr{M}(\Delta) \ni v \rightarrow \phi_{f}(v)=\int_{\Delta} f \mathrm{~d} v \in \mathbb{R}^{N} .
$$

$\phi_{f}$ is clearly linear and $\sigma(\mathscr{M}(\Delta), \mathscr{C}(\Delta))$-continuous and therefore $\phi_{f}(\mathscr{P}(\Delta))$ is convex and compact. Furthermore, $f(z)=\int_{\Delta} f \mathrm{~d} \delta_{z}$ implies that

$$
f(\Delta) \subset \phi_{f}(\mathscr{P}(\Delta)) \text {. }
$$

We will present the complete proof in two steps.

Step 1. The result holds for $N \quad 1$ with $K \quad 1$. Indeed, in such a case take

$$
m=\operatorname{Min}\{f(z) ; z \in \Delta\} \leqslant \operatorname{Max}\{f(z) ; z \in \Delta\}=M .
$$

Since $f(\Delta)$ is connected we have that

$$
f(\Delta)=[m, M] .
$$

Moreover

$$
m=\int_{\Delta} m \mathrm{~d} v \leqslant \int_{\Delta} f \mathrm{~d} v \leqslant \int_{\Delta} M \mathrm{~d} v=M
$$

holds for every $v \in \mathscr{P}(\Delta)$, from where (20) leads to the existence of $z_{v} \in \Delta$ with $f\left(z_{v}\right)=\int_{\Delta} f \mathrm{~d} v$ for every $v \in \mathscr{P}(\Delta)$.

Step 2. The result holds for every $N \in \mathbb{N}$. Indeed, since $\phi_{f}(\mathscr{P}(\Delta))$ is convex and compact we have that

$$
\phi_{f}(\mathscr{P}(\Delta))=\operatorname{Co}\left(\operatorname{ext}\left(\phi_{f}(\mathscr{P}(\Delta))\right)\right) .
$$

Thus, for each $v \in \mathscr{P}(\Delta)$ there exists a finite set $\left\{u_{1}, u_{2}, \ldots, u_{K}\right\} \subset \operatorname{ext}\left(\phi_{f}(\mathscr{P}(\Delta))\right)$ generating $\int_{\Delta} f \mathrm{~d} v$ as a linear convex combination

$$
\int_{\Delta} f \mathrm{~d} v=\sum_{h 1}^{K} t_{h} u_{h}
$$

It is sufficient to see that every element in the set $\left\{u_{1}, u_{2}, \ldots, u_{K}\right\}$ above takes the form $u_{k} f\left(z^{k}\right), z^{k} \in \Delta$, so fix $u \in \operatorname{ext}\left(\phi_{f}(\mathscr{P}(\Delta))\right)$ and let us prove the latter expression. From the previous lemma there exists a linear function $L$ : $\mathbb{R}^{N} \mapsto \mathbb{R}$ with

$$
L(u)>L(w)
$$

for every $w \in \phi_{f}(\mathscr{P}(\Delta)), w \neq u$. Obviously,

$$
\int_{\Delta}(L \circ f) \mathrm{d} v=L\left(\int_{\Delta} f \mathrm{~d} v\right)
$$

for every $v \in \mathscr{P}(\Delta)$, so

$$
\int_{\Delta}(L \circ f) \mathrm{d} v_{u}=L(u)
$$

for those $v_{u} \in \mathscr{P}(\Delta)$ with $\int_{\Delta} f \mathrm{~d} v_{u}=u$ (whose existence follows from $u \in \phi_{f}(\mathscr{P}(\Delta))$ ). According to the results stated in Step 1 there exists $z \in \Delta$ with

$$
L(f(z))=(L \circ f)(z)=\int_{\Delta}(L \circ f) \mathrm{d} v_{u}=L(u)
$$

from where, bearing in mind that $f(z) \in f(\Delta) \subset \phi_{f}(\mathscr{P}(\Delta))$ (see (19) and (21)), we have that $f(z) \quad u$.

Theorem 7. With the notations above let $(\lambda, \xi)$ be a extreme point of $\mathscr{A}_{0}$ and let $(\lambda, \xi, v)$ be a solution of (17) and a extreme point of $\mathscr{A}$. Then, there exist $\left\{z^{1}, z^{2}, \ldots, z^{K}\right\} \subset \Delta$ and a linear convex combination 


$$
\sum_{k=1}^{K} t_{k} \delta_{z^{k}} t_{1}, t_{2}, \ldots, t_{K} \geqslant 0, \sum_{k 1}^{K} t_{k}=1
$$

such that $\left(\lambda, \xi, \sum_{k}^{K}{ }_{1} t_{k} \delta_{z^{k}}\right)$ also solves (17) and is a extreme point of $\mathscr{A}$. Furthermore, if $K^{*}$ is the number of strictly positive components of $(\lambda, \xi)$ then $K^{*}+K \leqslant r+s+1$.

Proof. The existence of $(\lambda, \xi, v)$ follows from Lemma 4, and Lemma 6 guarantees the existence of $\sum_{k}^{K} t_{k} \delta_{z^{k}}$ with

$$
\sum_{i=1}^{\gamma} \alpha_{i}\left(\int_{\Delta_{i}} E\left(y_{h} z_{i}\right) \mathrm{d} v_{i}\right)=\sum_{i=1}^{\gamma} \alpha_{i}\left(\int_{\Delta_{i}} E\left(y_{h} z_{i}\right) \mathrm{d}\left(\sum_{k 1}^{K} t_{k} \delta_{z^{k}}\right)\right),
$$

$h \quad 1,2, \ldots, n$. Thus, $\left(\lambda, \xi, \sum_{k}^{K} t_{k} \delta_{z^{k}}\right) \in \mathscr{A}$ and we can assume that $t_{k} \neq 0, k \quad 1,2, \ldots, K$. If $\left(\lambda, \xi, \sum_{k}^{K} t_{k} \delta_{z^{k}}\right)$ is not a extreme point of $\mathscr{A}$ then $\sum_{k}^{K}{ }_{1} t_{k} \delta_{z^{k}}$ can be replaced by a new linear convex combination $\sum_{k}^{K}{ }_{1} \tau_{k} \delta_{z^{k}}$ such that $\left(\tau_{k}\right)_{k}^{K}$ is a extreme point of the set composed of those $\left(\tilde{\tau}_{k}\right)_{k}^{K}$ with non-negative components, $\sum_{k}^{K}{ }_{1} \tau_{k}=1$ and

$$
\sum_{i=1}^{\gamma} \alpha_{i}\left(\int_{\Delta_{i}} E\left(y_{h} z_{i}\right) \mathrm{d} v_{i}\right)=\sum_{i=1}^{\gamma} \alpha_{i}\left(\int_{\Delta_{i}} E\left(y_{h} z_{i}\right) \mathrm{d}\left(\sum_{k=1}^{K} \tilde{\tau}_{k} \delta_{z^{k}}\right)\right) .
$$

Now it is easy to see that $\left(\lambda, \xi, \sum_{k}^{K} \tau_{k} \delta_{z^{k}}\right)$ is a extreme point of $\mathscr{A}$.

Finally, it only remains to see that $K^{*}+K \leqslant n+1$ (we still denote by $K$ the number of nun-null components of $\left(\tau_{k}\right)_{k=1}^{K}$ ). Since $\left(\lambda, \xi, \sum_{k=1}^{K} \tau_{k} \delta_{z^{k}}\right) \in \mathscr{A}$ one has

$$
\begin{aligned}
& \left.\begin{array}{ll}
\sum_{j 1}^{m} E\left(q_{j} y_{h}\right) \lambda_{j}-\sum_{k 1}^{K} \tau_{k}\left[\int_{\Delta}\left(\sum_{i 1}^{\gamma} \alpha_{i} E\left(y_{h^{2}} z\right)\right)\right] \mathrm{d} \delta_{z^{k}}=0, & h=1,2, \ldots, r \\
\sum_{j 1}^{m} E\left(q_{j} y_{h}\right) \lambda_{j}-\xi_{h}-\sum_{k 1}^{K} \tau_{k}\left[\int_{\Delta}\left(\sum_{i 1}^{\gamma} \alpha_{i} E\left(y_{h^{2}} z\right)\right)\right] \mathrm{d} \delta_{z^{k}}=0, & h=r+1, \ldots, r+s
\end{array}\right\} . \\
& \sum_{k 1}^{K} \tau_{k}=1 \\
& \lambda \geqslant 0, \xi \geqslant 0, \tau \geqslant 0
\end{aligned}
$$

If one fixes the whole set of parameters in the system above except $(\lambda, \xi)$ and $\tau=\left(\tau_{k}\right)_{k}^{K}$ we already know that there is a solution in the unknown $(\lambda, \xi, \tau)$. Moreover, since our concrete $(\lambda, \xi, \tau)$ is a extreme point of $\mathscr{A}$ it is also a extreme point of the set of solutions of the system above. Hence, $(\lambda, \xi, \tau)$ is a Basic Feasible Solution (Anderson and Nash, 1987) and whence it cannot have more than $n+1$ (number of equations) strictly positive components.

Remark 3. Notice that the necessary and sufficient optimality conditions (15) may be simplified by using the latter theorem.

Remark 4 (Simplex-like Algorithm). Anderson and Nash (1987) presented a simplex-like algorithm that solves some semiinfinite linear optimization problems. Since their approach does not exactly fit our problem (17), we will adapt the method. However, we will just present the algorithm without proofs, because they are quite similar to those provided by the authors above.

Step 1. Fix a initial Basic Feasible Solution (extreme point) $\left(\lambda, \xi, \sum_{k=1}^{K} t_{k} \delta_{z^{k}}\right)$ satisfying the conditions stated in Theorem 7. In order to find this first Basic Feasible Solution $(B F S)$ one can follow those procedures indicated in Anderson and Nash (1987)..$^{5}$

Consider the matrix of System (22) above

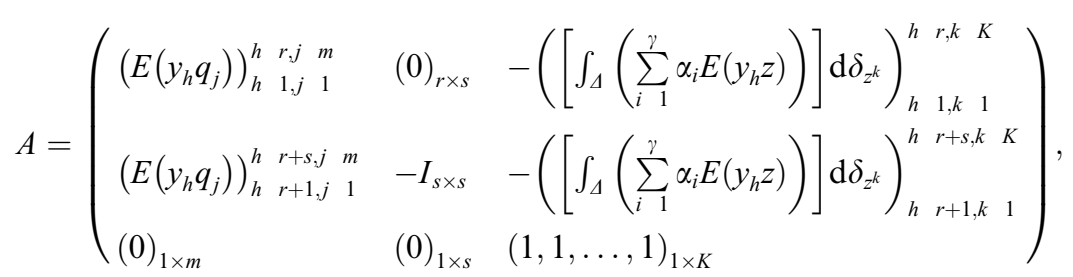

whose dimensions equal $(n+1) \times(m+s+K)$. Consider a sub-matrix $B$ taking the columns of $A$ associated with non-null elements of $\left(\lambda, \xi, \sum_{k}^{K} t_{k} \delta_{z^{k}}\right)$. Theorem 7 guarantees that $B$ has less than $(n+1)$ columns or exactly $(n+1)$ columns. In the

${ }^{5}$ For instance, there exists a two phases like method. 
first case this $B F S$ is said to be degenerated and non-degenerated in the second one. If we were facing degeneration then we would add some columns of $A$ so as to reach a square and regular $B$. Consider finally the row matrix

$$
c=\left(-b_{1},-b_{2}, \ldots,-b_{m}, 0, .0, \ldots, 0\right)
$$

with $(m+s+K)$ columns and the row matrix $c_{B}$ with $(n+1)$ columns that is obtained taking from $c$ those elements associated with the columns of $A$ composing $B$ (henceforth, basic columns).

Step 2. Compute the matrices

$$
B^{-1} A \text { and } c_{B} B^{-1} A-c \text {. }
$$

It is trivial to show that those columns of $B{ }^{1} A$ associated with the basic columns generate the identity matrix and, consequently, those elements of $c_{B} B{ }^{1} A-c$ associated with the basic columns must vanish. In particular, all of the elements associated with $\sum_{k 1}^{K} t_{k} \delta_{z^{k}}$ must vanish.

Consider the function

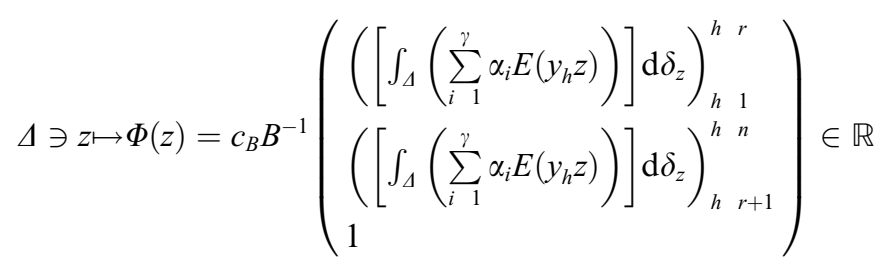

We can extend the matrix $B{ }^{1} A$ by adding a new row and a two new columns. We obtain the simplex tableau

$$
\left(\begin{array}{rrr} 
& c_{B} B^{-1} A-c . & -\sum_{j 1}^{m} b_{j} \lambda_{j} \\
\left(\begin{array}{l}
\left(j_{l}\right)_{j_{l}} \\
\left(h_{l}\right)_{h_{l}} \\
\left(z_{k_{l}}\right)_{k_{l}}
\end{array}\right) & B^{-1} A, & B^{-1}\left(\begin{array}{l}
0 \\
\cdot \\
\cdot \\
1
\end{array}\right)
\end{array}\right)
$$

indicating those variables that are basic (first column), their values (last column) and the objective level.

Optimality criterion. If there are no negative elements in $c_{B} B{ }^{1} A-c$ and

$$
\Phi(z) \geqslant 0
$$

for every $z \in \Delta$ then $\left(\lambda, \xi, \sum_{k}^{K} t_{1} t_{k} \delta_{z^{k}}\right)$ solves (17). The algorithm ends here. Otherwise we must go to Step 3.

Step 3. Scenario 1. Assume that $c_{B} B{ }^{1} A-c$ contains a negative element. It is associated with a non-basic column of $A$,

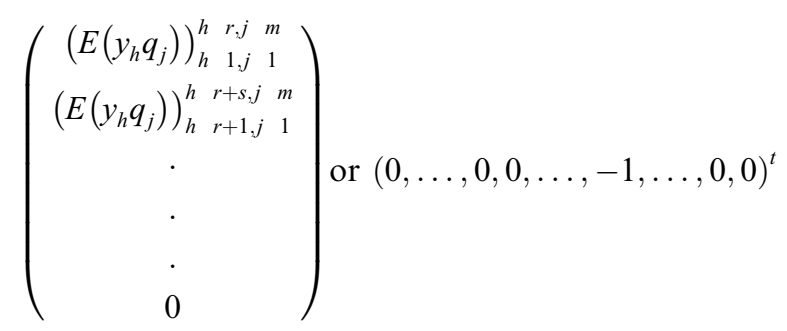

related to a non-basic variable $\lambda_{j_{0}}$ or $\xi_{h_{0}}$ that will become basic in a new iteration of the algorithm. Denote by $A_{0}$ the column above and compute the column matrix (which is a column of $B{ }^{1} A$ )

$$
\left(\eta_{1}, \eta_{2}, \ldots, \eta_{n+1}\right)^{t}=B^{-1} A_{0} .
$$

It may be easily proved that if there were no positive elements in $B{ }^{1} A_{0}$ then problem (17) would be unbounded, and (9) would imply that (13) should be unfeasible. Suppose that $B{ }^{1} A_{0}$ contains positive elements and take

$$
\eta=\operatorname{Min}\left\{\left\{\frac{\lambda_{j_{l}}}{\eta_{l}} ; \eta_{l}>0\right\} \cup\left\{\frac{\xi_{h_{l}}}{\eta_{l}} ; \eta_{l}>0\right\} \cup\left\{\frac{t_{k_{l}}}{\eta_{l}} ; \eta_{l}>0\right\}\right\},
$$

with the obvious notations $\left(\left(\left(\lambda_{j_{l}}\right),\left(\xi_{h_{l}}\right),\left(t_{k_{l}}\right)\right)=B^{-1}(0,0, \ldots, 1)^{t}\right.$ is in the last column of $(24)$ and represents here the "old" or non-optimal basic feasible solution). The minimum value is attained at the element representing the variable that will give up being basic in the next iteration. Therefore we have modified the set a basic variables, and we can solve System (22) so as to obtain a new BFS. The objective value has been improved in the new BFS and we have to go back to Step 1. 
However, some computations can be accelerated if one proceeds as follows: Call "pivot" $P$ to that element of $B{ }^{1} A$ in the position where the minimum (27) above is reached. Update the row of $B{ }^{1} A$ and Tableau (24) containing the pivot by dividing the whole row by $P$ (one will obtain the new value 1 instead of $P$ ). Update $c_{B} B{ }^{1} A-c$ and the remaining rows of $B^{1} A$ and (24) by subtracting from the old one the new row containing the pivot multiplied by $P_{l}$, element in the corresponding row and in the same column as the pivot. Modify the subscripts included in the first column of (24) so as to reflect the new basic variables. We can now go back to Step 2 and we have already updated $\left(\lambda, \xi, \sum_{k=1}^{K} t_{k} \delta_{z^{k}}\right), B{ }^{1} A, c_{B} B{ }^{1} A$ $c$ and (24).

Step 3. Scenario 2. Assume that $c_{B} B{ }^{1} A$-c does not contain any negative element but there exists $\tilde{z} \in \Delta$ with $\Phi(\tilde{z})<0$. Obviously, $\tilde{z}$ does not belong to the set $\left\{z^{1}, z^{2}, \ldots, z^{K}\right\}$. If possible, choose $\tilde{z}$ so as to solve

$$
\operatorname{Min}\{\Phi(z) ; z \in \Delta\} .
$$

Otherwise choose an arbitrary $\tilde{z}$ making negative the value of $\Phi . \tilde{z}$ will belong to the next $B F S$ and we have to determine to variable to leave the basis. In order to do that we follow the same way as in the previous scenario. Thus, let

$$
A_{0}=\left(\begin{array}{l}
\left(\left[\int_{\Delta}\left(\sum_{i 1}^{\gamma} \alpha_{i} E\left(y_{h} \tilde{z}\right)\right)\right] \mathrm{d} \delta_{z}\right)_{h}^{h r} \\
\left(\left[\int_{\Delta}\left(\sum_{i 1}^{\gamma} \alpha_{i} E\left(y_{h} \tilde{z}\right)\right)\right] \mathrm{d} \delta_{z}\right)_{h r+1}^{h}{ }_{h} \\
1
\end{array}\right)
$$

compute (26), and the value $\eta$ of (27) will indicate the variable to stop being basic. Now we can solve System (22) so as to obtain a new $B F S$ and we have to go back to Step 1. We can simplify some computations if we update $\left(\lambda, \xi, \sum_{k}^{K} t_{k} \delta_{z^{k}}\right)$, $B^{1} A, c_{B} B{ }^{1} A-c$ and (24) by proceeding as in the previous case. If so, it is worth to extend "the old $B{ }^{1} A, c_{B} B{ }^{1} A-c$ and (24)" before the computations by adding the new column

$$
\left.\left(\begin{array}{c}
\left(\left[\int_{\Delta}\left(\sum_{i 1}^{\gamma} \alpha_{i} E\left(y_{h} \tilde{z}\right)\right)\right] \mathrm{d} \delta_{z}\right)_{h \quad 1}^{h r} \\
\left(\left[\int_{\Delta}\left(\sum_{i=1}^{\gamma} \alpha_{i} E\left(y_{h} \tilde{z}\right)\right)\right] \mathrm{d} \delta_{z}\right)_{h r+1}^{h{ }^{-1}} \\
1
\end{array}\right)\right) .
$$

Final Note. If the optimality criterion of Step 2 does not hold and we go to the second scenario of Step 3 then we should choose $\tilde{z} \in \Delta$ such that $\Phi(\tilde{z})$ were as small (negative) as possible (we should even solve problem (28), in order to achieve a good convergence of the algorithm to the solution). Thus, this pivotal step might be important, but $\Phi$ being linear in the $z$ variable (see (23)) one can check the value of $\Phi$ in the extreme points of $\Delta$ in order to get an element $\tilde{z}$ as close as possible to the solution of (28) Anyway, it is worth to recall that the objective function does not directly depend on the $v$ variable, whose unique effect is on the constraints of the problem. In practice, as will be said in Section 7, the convergence of the algorithm is very fast.

\section{Some significant risk functions}

This section will be devoted to illustrate several examples of risk functions satisfying (1) i.e., such that the developed methodology applies. All the examples here presented have been already treated in the literature, so our unique purpose is to show how general our methods may be. Thus, we will not be exhaustive and will only summarize the most important properties of some significant risk functions.

All the examples are positively homogeneous and convex functions. Thus, if we maximize a generalized Sharpe ratio $(G S R)$, quotient between the expected risk premium and the risk excess with respect to the risk-free security, then we will obtain a special strategy that composes the efficient portfolios in a "new risk/return framework". Indeed, once we fix the risk function, we can outperform every feasible portfolio by adequately combining the riskless asset and the strategy maximizing the GSR. This property is similar to that well known in the CAPM model, where the risk function is the standard deviation and efficiency is achieved if and only if one diversifies between the riskless security and the Market Portfolio. Obviously, the maximization of the $G S R$ is equivalent to the minimization of the risk level under an appropriate linear constraint on the expected risk premium, i.e., the here proposed methodology applies. In some sense one can interpret that we are constructing a "new index" that solves those problems generated by asymmetries and heavy tails. 
Example 1 (Standard Deviation). The standard deviation $\sigma_{2}$ is the dispersion measure used in classical Portfolio Selection Theory and the Equilibrium Models $C A P M$ and $A P T$. It is defined over the Banach space $L^{2}$. According to the results of Rockafellar et al. (2006a), expression (1) holds if (see (2) and (3))

$$
\tilde{\Delta}^{\sigma_{2}}=\Delta^{\sigma_{2}}-\{1\}=\left\{z \in L^{2} ; E(z)=1,\|z-1\|_{2} \leqslant 1\right\}-\{1\}=\left\{z \in L^{2} ; E(z)=0,\|z\|_{2} \leqslant 1\right\},
$$

which is a $\sigma\left(L^{2}, L^{2}\right)$-compact set because it is bounded (recall the Alaoglu's Theorem). The related risk measure also satisfies (1) if we take the compact space $\Delta^{\sigma_{2}}$. Since, for a general probability space $(\Omega, \mathscr{F}, \mu), \Delta^{\sigma_{2}}$ contains non-positive random variables, the risk measure is not decreasing, and therefore it is not coherent in the sense of Artzner et al. (1999) (see Rockafellar et al., 2006a). This is a shortcoming because risk measures try to represent capital requirements, and higher pay-offs might lead to higher initial capital reserves. As already said, the deviation measure $\sigma_{2}$ is not compatible with the second order stochastic dominance (SOSD) if asymmetric returns are involved Ogryczak and Ruszczynski (1999).

Example 2 (Usual dispersions). The $p$-deviation is given by $L^{p} \ni y \mapsto \sigma_{p}(y)=\|(y-E(y))\|_{p}=\left[E|y-E(y)|^{p}\right]^{1 / p} \in \mathbb{R}$, where $p \in[1, \infty)$. Since $\|\cdot\|_{q}$ is the dual norm of $\|\cdot\|_{p}$ we have that $\|y\|_{p}=\operatorname{Sup}\left\{E(y z) ; z \in L^{q},\|z\|_{q} \leqslant 1\right\}$ holds for every $y \in L^{p}$. Hence,

$$
\begin{aligned}
\sigma_{p}(y) & =\|(y-E(y))\|_{p}=\operatorname{Sup}\left\{E[(y-E(y)) z] ; z \in L^{q},\|z\|_{q} \leqslant 1\right\}=\operatorname{Sup}\left\{E(y z)-E(y) E(z) ; z \in L^{q},\|z\|_{q} \leqslant 1\right\} \\
& =\operatorname{Sup}\left\{E[y(z-E(z))] ; z \in L^{q},\|z\|_{q} \leqslant 1\right\}=\operatorname{Sup}\left\{E(y(-z)) ; z=E\left(z^{\prime}\right)-z^{\prime}, z^{\prime} \in L^{q},\left\|z^{\prime}\right\|_{q} \leqslant 1\right\} \\
& =\operatorname{Sup}\left\{E(y(-z)) ; z \in \tilde{\Delta}^{\sigma_{p}}\right\} .
\end{aligned}
$$

Since $L^{q} \ni z \mapsto E(z)-z \in L^{q}$ is $\sigma\left(L^{q}, L^{p}\right)$-continuous and the unit ball of $L^{q}$ is $\sigma\left(L^{q}, L^{p}\right)$-compact (Alaoglu's Theorem), $\tilde{\Delta}^{\sigma_{p}}$ is $\sigma\left(L^{q}, L^{p}\right)$-compact, i.e, expression (1) and the theory developed in this paper applies.

As in the previous example, $\Delta^{\sigma_{p}}=\tilde{\Delta}^{\sigma_{p}}+\{1\}$ contains non-positive random variables and, therefore, the associated expectation bounded risk measure is non-decreasing and non-coherent in general.

The absolute deviation $\sigma_{1}(y) \quad E|y-E(y)|, y \in L^{1}$, presents a significant property. Indeed, it is always compatible with the SOSD (Ogryczak and Ruszczynski, 1999). Moreover, if we introduce a minor distortion and use $\frac{\sigma_{1}(y)}{2}$ rather than $\sigma_{1}(y)$, then the associated risk measure $\frac{\sigma_{1}(y)}{2}-E(y)$ becomes coherent (Rockafellar et al., 2006a).

The $p$-semi-deviation is given by $L^{p} \ni y \mapsto \sigma_{p}(y)=\left\|(y-E(y))_{-}\right\|_{p} \in \mathbb{R}$ where

$$
(y-E(y))_{-}=\left\{\begin{array}{ll}
0 & \text { if } y \geqslant E(y) \\
E(y)-y & \text { otherwise }
\end{array} .\right.
$$

It is an interesting dispersion measure in portfolio choice theory because it only considers those deviations with respect to the expected pay-off with negative effect on the investor final wealth. Once again it may be represented by expression (1) (Rockafellar et al., 2006a) and the associated risk measure is expectation bounded and coherent (Rockafellar et al., 2006a), and for $p \quad 1$ or $p \quad 2$ it is compatible with the SOSD (Ogryczak and Ruszczynski, 1999).

Example 3 (Conditional Value at Risk). The conditional value at risk ( $C V a R$ or $\left.C V a R_{\mu_{0}}\right)$, defined on $L^{1}$, is becoming a very important risk measure, quite analyzed in theoretical studies and quite used by traders in practice. It is coherent and Rockafellar et al. (2006a) stated that it is expectation bounded and can be represented with

$$
\Delta^{C V a R}=\left\{z \in L^{\infty} ; 0 \leqslant z \leqslant \frac{1}{\mu_{0}}, E(z)=1\right\} \subset L^{\infty}
$$

$\mu_{0} \in(0,1)$ being the level of confidence. $\Delta^{C V a R}$ is convex and $\sigma\left(L^{\infty}, L^{1}\right)$-compact. The extreme set of $\Delta^{C V a R}$ is important to make it easier to deal with Condition (25) and problem (28). It is composed of those random variables taking the form $\left(1 / \mu_{0}\right) \chi_{\Omega_{0}}, \Omega_{0} \in \mathscr{F}, \mu\left(\Omega_{0}\right) \quad \mu_{0}$. The deviation associated with $C V a R$ can also be treated with our methodology since it is represented by the convex compact set

$$
\tilde{\Delta}^{\text {CVaR }}=\left\{z \in L^{\infty} ;-1 \leqslant z \leqslant \frac{1}{\mu_{0}}-1, E(z)=0\right\} \subset L^{\infty} .
$$

Despite its growing importance, $C V a R$ is not compatible with the $S O S D$, as we will see in Example 5.

Example 4 (Value at Risk). It is well-known that the value at risk $(\mathrm{VaR})$ is also very important in practice, despite it is neither coherent nor expectation bounded, and it does not respect the SOSD. Since VaR cannot be represented according to (1), our methodology does not apply either. However, it is worth to point out that, under appropriate assumptions, some modifications of problem (5) would provide a linear vector optimization problem in Banach spaces equivalent to problem 
(4). Hence, the methodology here presented may be adapted so as to address minimum $V a R$ related topics (linear programming vector problems in general Banach spaces are analyzed, for instance, in (Balbás and Heras, 1993)).

Example 5 (Distortion Functions). Let us analyze some risk functions satisfying a collection of "suitable properties" (coherent and expectation bounded risk measures satisfying (1) and such that they and their deviations are compatible with the $S O S D)$.

Wang (2000) considers a non-decreasing function $g:[0,1] \mapsto[0,1]$ with $g(0) \quad 0$ and $g(1) \quad 1$, and a general risk measure with the "heuristic form"

$$
R_{g}(y)=\int_{0}^{1} \operatorname{VaR}_{t}(y) \mathrm{d} g(t)
$$

where $\operatorname{VaR}_{t}(y)$ stands for Value at Risk of the random variable $y$ with $t$ as the confidence level. If $g$ is continuous in $[0,1]$ and piecewise differentiable in $(0,1)$ then the expression above gives

$$
R_{g}(y)=\int_{0}^{1} \operatorname{VaR}_{t}(y) g^{\prime}(t) \mathrm{d} t .
$$

A special important case is

$$
g(t)= \begin{cases}\left(1 / \mu_{0}\right) t, & t \leqslant \mu_{0} \\ 1, & t \geqslant \mu_{0}\end{cases}
$$

that leads to $\mathrm{CVaR}_{\mu_{0}}$. Wang justifies his proposal by several reasons. Among them, he shows simple numerical examples illustrating that $C V a R$ might present some drawbacks in particular practical problems. The intuitive idea of Wang is formalized in Whirch and Hardy (2001) where it is established that $R_{g}$ is compatible with the SOSD if and only if $g$ is strictly concave, case in which $R_{g}$ is also coherent.

There are many risk measures given by (29) satisfying a set of desirable conditions for which our methodology applies. For example, suppose that $g$ is two times continuously differentiable in $[0,1]$ and with strictly negative second derivative in $(0,1)$. Then $R_{g}$ is defined in $L^{1}$, compatible with the SOSD, and it may be stated that (1) holds if $\Delta^{g}$ is the weakly ${ }^{*}$-closed convex hull of

$$
\{0\} \cup\left\{z \in L^{\infty} ; z(\omega)=g^{\prime}[\mu(y \leqslant y(\omega))], y \in L^{1}\right\} .
$$

Since $g^{\prime}$ is non-negative and bounded from above $R_{g}$ is coherent (Rockafellar et al., 2006a) and expectation bounded, and $\Delta^{g}$ is $\sigma\left(L^{\infty}, L^{1}\right)$-compact, that is, $R_{g}$ satisfies the whole set of "ideal properties" and the methodology of this paper applies. An important example in Actuarial Sciences is the "Dual Power Transform", generated by

$$
g_{x}(t)=1-(1-t)^{x}, \quad x \geqslant 2 .
$$

Wang also proposed the distorting function $g(t) \quad \Psi\left(a+\Psi^{1}(t)\right)$, with $a>0$ and $\Psi$ denoting the cumulative function of the standard normal distribution. Then $R_{g}$ is defined in $L^{2}$, compatible with the $S O S D$, and it may be stated that (1) holds if $\Delta^{g}$ is the weakly closed convex hull of

$$
\{0\} \cup\left\{z \in L^{2} ; z(\omega)=g^{\prime}[\mu(y \leqslant y(\omega))], y \in L^{2}\right\} .
$$

Since $g^{\prime}$ is non-negative and belongs to $L^{2}[0,1]$ (when $[0,1]$ is endowed with the Measure of Lebesgue), $\Delta^{g}$ is $\sigma\left(L^{2}, L^{2}\right)$-compact and (according to (Rockafellar et al., 2006a)) $R_{g}$ is coherent and expectation bounded.

\section{Numerical example}

Let us present a numerical example illustrating the performance of the proposed methodology in practice. We will consider a real database containing weekly prices from June 27th, 2003 to September 22nd 2006. ${ }^{6}$ There are eight involved securities: A riskless asset and seven risky ones. They are $I E$ (international equity), $S E$ (Equity, small companies), $E E$ (Equity, emerging regions), FI (Fixed Income), EFI (Fixed Income, emerging regions and High Yield), a Hedge Fund denoted $R 1$, and a final portfolio COM reflecting a combination of commodity-linked future contracts.

We will deal with a Portfolio Choice problem and the risk level will be given by the Wang measure and the Conditional Value at Risk, respectively. As said above, $C V a R$ is becoming very used by practitioners, despite it is not compatible with the SOSD. Furthermore, the literature has shown that it may be quite complex to optimize $C V a R$ in practice, mainly for some kind of securities (see, amongst many others, Alexander et al., 2006). On the other hand, as far as we know, there are

\footnotetext{
${ }^{6}$ The authors thank "Welzia Management SGIIC SA" for the database.
} 
no empirical papers optimizing the Wang measure, despite it is coherent, expectation bounded and compatible with the SOSD.

Consider problem

$$
\left.\begin{array}{l}
\operatorname{Min} \rho(u) \\
E\left(y q_{j}\right) \leqslant b_{j} \quad j=1,2
\end{array}\right\}
$$

where $\rho$ equals $C \operatorname{VaR}$ (we will provide the value of $\mu_{0}$ ) or the Wang measure (a 1.65, according to the (Wang, 2000), suggestion). The first constraint is related to the portfolio expected return that must outperform the weekly return $r \quad 0.001$. Thus $q_{1} \quad(-1, \ldots,-1)$ and $b_{1} \quad-(1+r) \quad-1.001$, so the restriction becomes $E(-y) \leqslant-(1+r)$. The second constraint imposes that we will not invest more than one Euro, and so this restriction becomes $E\left(y q_{2}\right) \leqslant 1\left(b_{2} \quad 1\right), q_{2}$ denoting the Stochastic Discount Factor (see (Cochrane, 2001), for further details on this concept). By standard financial arguments it is easy to show that the absence of arbitrage implies that problem (30) is bounded (Cochrane, 2001). Moreover, the Slater Qualification of Assumption 1 also holds since $r$ is small and there are several securities whose expected weekly returns are larger than $r$.

We have been drawing on the presented simplex-like algorithm under different assumptions. For the sake of simplicity we will just report the numerical solutions (optimal portfolios) since there is nothing significant related to the application of the algorithm. Despite the linear optimization problem involves infinite-dimensional spaces, the objective function only depends on the finite-dimensional dual variable $\lambda$ (see problem (17)), so the convergence of the algorithm is really fast. We just developed a Math-Lab code and the computation time was never longer than 6 min. $^{7}$

Strategy 1.1. We do not incorporate the riskless asset and impose $x_{k} \geqslant 0, k \quad 1, \ldots, 7$. We have to adapt (30) so as to obtain an expression similar to (13). Then, if $\rho$ is the measure of Wang we solve (17) and get

$$
\begin{aligned}
& \lambda_{1}=2.739488, \quad \lambda_{2}=1.7130599 \\
& \xi_{1}=0.0004, \quad \xi_{5}=0.0016, \quad \xi_{k}=0, \\
& k=2,3,4,6,7 \\
& t_{1}=0.1628, \quad t_{2}=0.1886, \quad t_{3}=0.6486
\end{aligned}
$$

The Complementary Slackness Conditions (16) and the constraints of (13) lead to

$$
\begin{aligned}
& \theta=-0.993920023 \\
& x_{2}=0.0500, \quad x_{3}=0.0100, \quad x_{4}=0.1062, \quad x_{5}=0.8000, \quad x_{6}=0.0338 \\
& x_{k}=0, \quad k=1,7
\end{aligned}
$$

Strategy 1.2. Under the conditions above, if $\rho \quad C V a R_{0.03}$ (i.e., $\mu_{0} \quad 0.03$ is the level of confidence) we get

$$
\begin{aligned}
& \lambda_{1}=2.686958046, \quad \lambda_{2}=1.680211521 \\
& \xi_{1}=0.0076, \quad \xi_{3}=0.0047, \quad \xi_{5}=0.0113, \xi_{k}=0, \quad k=2,4,6,7 \\
& t_{1}=0.2540, \quad t_{2}=0.2677, \quad t_{3}=0.4783 .
\end{aligned}
$$

Besides

$$
\begin{aligned}
& \theta=-0.995607081 \\
& x_{2}=0.0500, \quad x_{4}=0.1500, \quad x_{6}=0.7442, \quad x_{7}=0.0558 \\
& x_{k}=0, \quad k=1,3,5
\end{aligned}
$$

is the primal solution.

Strategy 1.3. Under the conditions above, if $\rho \quad C V a R_{0.0592}$ ( i.e., $\mu_{0} \quad 0.0592 \quad 5.92 \%$ is the level of confidence)

$$
\begin{aligned}
& \lambda_{1}=4.463566171, \quad \lambda_{2}=3.7728751 \\
& \xi_{1}=0.0008, \quad \xi_{5}=0.0006, \quad \xi_{k}=0, \quad k=2,3,4,6,7 \\
& t_{1}=0.0581, \quad t_{2}=0.2692, \quad t_{3}=0.1682, \quad t_{4}=0.5045
\end{aligned}
$$

\footnotetext{
${ }^{7}$ Recall that we are testing the performance of the Simplex like algorithm, rather than the performance of the selected risk functions.
} 
The primal solution becomes

$$
\begin{aligned}
& \theta=-0.997226608 \\
& x_{2}=0.0500, \quad x_{3}=0.0199, \quad x_{4}=0.3000, \quad x_{6}=0.6301 \\
& x_{k}=0, \quad k=1,5,7
\end{aligned}
$$

Strategy 2.1. Let us consider the assets $S_{1} \quad E E, S_{2} \quad F I, S_{3} \quad R 1, S_{4} \quad C O M$, and the riskless asset $S_{5}$. In order to simplify take a null risk-free rate. Impose $x_{k} \geqslant 0, k \quad 1, \ldots, 5$. For the Wang measure we get

$$
\begin{aligned}
& \lambda_{1}=2.68386099, \quad \lambda_{2}=1.67825611 \\
& \xi_{5}=0.0099, \quad \xi_{k}=0, \quad k \neq 5 \\
& t_{1}=0.3950, \quad t_{2}=0.4469, \quad t_{3}=0.1581
\end{aligned}
$$

and

$$
\begin{aligned}
& \theta=-0.991837854 \\
& x_{1}=0.0168, \quad x_{2}=0.1500, \quad x_{3}=0.7332, \quad x_{4}=0.100, \quad x_{5}=0
\end{aligned}
$$

Strategy 2.2. Under the assumptions of the latter strategy, $\rho \quad C V a R_{0.03}$ leads to

$$
\begin{aligned}
& \lambda_{1}=2.685878, \quad \lambda_{2}=1.6795367 \\
& \xi_{3}=0.0024, \quad \xi_{4}=0.0006 \\
& t_{1}=0.3610, \quad t_{2}=0.6390
\end{aligned}
$$

and

$$
\begin{aligned}
& \theta=-0.995584188 \\
& x_{1}=0.0528, \quad x_{2}=0.4000, \quad x_{3}=0.5472, \quad x_{k}=0, \quad k=4,5
\end{aligned}
$$

\section{Conclusions}

Capital requirements of Financial Institutions, fat-tailed return distributions and asymmetric returns have provoked a growing interest in modern risk analysis. Researchers, regulators and practitioners are sharing in the development of new methods measuring risk levels of investment strategies.

Portfolio optimization and optimal hedging are complex in practice when general risk functions are involved. These functions are usually non-differentiable and it is also complex to apply the properties of Convex (or convex-like) Programming. Thus, the development of appropriate optimality conditions and algorithms is becoming an important topic.

Due to the Representation Theorems of Risk Measures we have transformed a (vector) Risk Minimization problem of Portfolio Choice Theory into a Minimax problem, and consequently, into a dual pair of Linear Programming problems between infinite-dimensional Banach spaces of continuous functions and inner regular $\sigma$-additive measures. Accordingly, necessary and sufficient optimality conditions have been established. They do not draw on the concept of subgradient, and some probability measures are involved as multipliers.

With respect to previous studies transforming portfolio choice problems into linear ones the present approach seems to reflect an important contribution. Indeed, the methodology is general enough and includes every expectation bounded or dispersion risk measure, as well as most of the coherent risk measures.

The dual problem usually becomes semi-infinite, allowing for a simplex-like algorithm leading to both primal and dual solutions. Thus, one has a practical method to obtain optimal strategies and their sensitivities. Furthermore, in the semiinfinite case the dual solution is achieved at a convex combination of Dirac deltas, allowing for new versions and interpretations of the optimality conditions. Despite the dual optimization problem involves infinite-dimensional spaces, the objective function only depends on a finite-dimensional subspace, so the practical convergence of the algorithm is really fast.

As said above, the theory applies for many coherent, expectation bounded and deviation risk measures and, therefore, it can be particularized to address those special cases we may be interested in.

\section{Acknowledgements}

Research partially supported by "Welzia Management SGIIC SA", "RD Sistemas SA", "Comunidad Autónoma de Madrid" (Spain), Grant s-0505/tic/000230, and "MEyC" (Spain), Grant SEJ2006-15401-C04-03. The authors thank the anonymous reviewers for their suggestions that have led to several improvements of the paper The usual caveat applies. 


\section{References}

Alexander, S., Coleman, T.F., Li, Y., 2006. Minimizing CVaR and VaR for a portfolio of derivatives. Journal of Banking \& Finance $30,538605$. Anderson, E.J., Nash, P., 1987. Linear Programming in Infinite dimensional Spaces. John Wiley \& Sons, New York.

Artzner, P., Delbaen, F., Eber, J.M., Heath, D., 1999. Coherent measures of risk. Mathematical Finance 9, 203228.

Balbás, A., Heras, A., 1993. Duality theory for infinite dimensional multiobjective linear programming. European Journal of Operational Research 68 , 379388.

Balbás, A., Romera, R., 2007. Hedging bond portfolios by optimization in Banach spaces. Journal of Optimization Theory and Applications 132 (1), 175 191.

Barber, J.R., Copper, M.L., 1998. A minimax risk strategy for portfolio immunization. Insurance: Mathematics and Economics 23 , 173177.

Benati, S., 2003. The optimal portfolio problem with coherent risk measure constraints. European Journal of Operational Research $150,572584$.

Cochrane, J.H., 2001. Asset Pricing. Princeton University Press.

Follmer, H., Schied, A., 2002. Convex measures of risk and trading constraints. Finance \& Stochastics 6, 429447.

Holmes, R.B., 1975. Geometrical functional analysis and its applications. In: Graduate Texts in Mathematics, vol. 24. Springer Verlag, New York Heidelberg.

Konno, H., Akishino, K., Yamamoto, R., 2005. Optimization of a long short portfolio under non convex transaction costs. Computational Optimization and Applications 32, 115132

Nakano, Y., 2004. Efficient hedging with coherent risk measure. Journal of Mathematical Analysis and Applications $293,345354$.

Ogryczak, W., Ruszczynski, A., 1999. From stochastic dominance to mean risk models: Semideviations and risk measures. European Journal of Operational Research 116, 3350.

Rockafellar, R.T., Uryasev, S., Zabarankin Generalized deviations in risk analysis, M., 2006a. Finance \& Stochastics $10,5174$.

Rockafellar, R.T., Uryasev, S., Zabarankin, M., 2006b. Optimality conditions in portfolio analysis with general deviations measures. Mathematical Programming Ser. B 108, 515540.

Ruszczynski, A., Shapiro, A., 2007. Optimization of risk measures. Mathematics of Operations Research, forthcoming.

Shimizu, K., Aiyoshy, E., 1980. Necessary conditions for Min Max problems and algorithms by a relaxation procedure. Transactions on Automatic Control 25, 6267.

Wang, S.S., 2000. A class of distortion operators for pricing financial and insurance risks. Journal of Risk and Insurance $67,1536$.

Whirch, J.L., Hardy, M.R., 2001. Distortion risk measures. Coherence and stochastic dominance. Unpublished Working Paper (http:// www.gloriamundi.org).

Young, M.R., 1998. A minimax portfolio selection rule with linear programming solution. Management Science 43, 673683.

Zalinescu, C., 2002. Convex Analysis in General Vector Spaces. World Scientific Publishing Co. 